\title{
Mass Filter Characteristic and Design Role of Quadrupole Mass Spectrometer for Radical Measurement in Low-pressure Plasmas
}

\author{
Received July 15, 2020; revised July 21, 2020; accepted July 22, 2020
}

Hee Jung Yeom ${ }^{a, b}$, Jang Jae Lee ${ }^{c}$, Si Jun Kimª, Young Seok Lee ${ }^{a}$, Chul hee Cho ${ }^{a}$, Jung Hyung Kim ${ }^{b}$, Hyo-Chang Lee ${ }^{b}$, and ShinJae You ${ }^{\mathrm{a}, *}$

\author{
aDepartment of Physics, Chungnam National University, Daejeon 34134, Republic of Korea \\ ${ }^{b}$ Korea Research Institute of Standards and Science, Daejeon 34113, Republic of Korea \\ Institute of Quantum System (IQS), Chungnam National University, Daejeon 34134, Republic of Korea
}

*Corresponding author E-mail: sjyou@cnu.ac.kr

\begin{abstract}
In this study, we confirmed through experiments that a noise signal appears in the low-mass region when measuring plasma using a quadrupole mass spectrometer. We conducted a numerical analysis to identify the cause of this occurrence. It was determined that high-energy ions generated in plasma are not filtered in the low quadrupole filter voltage region owing to the high velocity of the ions. We thus detected the signal in the low-mass region based on the stability area. In this paper, we identify through numerical analysis the quadrupole filter length and the radius of the incident ion beam that effectively reduce low-mass noise.
\end{abstract}

Keywords: Quadrupole mass filter, Radical, Plasma

\section{Introduction}

Recently, it has become very important to measure the gas composition and the partial pressure in industrial semiconductor and display processing, such as etching, deposition, and ashing [1-5]. Among many possible diagnostic methods, the mass spectrometer is an investigative tool that offers many advantages for analyzing reactive plasmas on account of its high sensitivity and mass resolution characteristics [3,6-9]. Many kinds of mass spectrometers exist, such as the time of flight mass spectrometer [10], magnetic sector mass spectrometer $[11,12]$, and quadrupole mass spectrometer (QMS) [13]. Among these, QMS is often used for measuring radicals in plasma owing to its low cost, relatively small size, and robustness $[8,14]$. When measuring radical species in plasma using QMS, potential problems may arise, such as low mass tailing [15], changed acceptance [16,17], and abnormal signals in the low-mass region [18]. Nevertheless, previous research has not determined whether such a problem actually occurs in plasma measurement.

In this study, we confirmed that an abnormal signal occurs in the low-mass region during plasma measurement. The cause of this occurrence was determined by numerical analysis. Through a numerical simulation of the quadrupole mass spectrometer, the transmission characteristics of the ions entering the quadrupole filter in accordance with the initial conditions, such as the preliminary velocity and initial position, were analyzed. Accordingly, the design conditions for the quadrupole filter length and ion beam radius in plasma measurement were studied.

\section{Experimental details}

A radical measurement was performed in capacitively coupled plasma. The chamber geometry, radio-frequency power feeding, and QMS (Stanford Research Systems, RGA 200) are shown in Fig. 1. The chamber is a cylindrical shape with an inner diameter of $340 \mathrm{~mm}$ and a distance of $50 \mathrm{~mm}$ from the top to the bottom of the electrode. A $13.56 \mathrm{MHz}$ power supply is connected to the bottom electrode through an L-type auto-matcher. The input power during the experiment was 0 and $500 \mathrm{~W}$. The gases utilized in the experiment were $\mathrm{Ar}$ and $\mathrm{C}_{4} \mathrm{~F}_{8}$ with a fixed $20 \mathrm{sccm}$ flow rate, respectively. A rotary pump and turbo-molecular pump were employed to maintain the background gas pressure of the processing chamber below $5 \times 10^{-6}$ Torr, which was measured using a Penning gauge. The experimental pressure was 10 mTorr. It was measured using a capacitive diaphragm gauge. The base pressure of the QMS was set below $2 \times 10^{-7}$ Torr using a $90 \mu \mathrm{m}$ diameter orifice and a differential pumping system.

Figure 2 shows the mass spectra of the $\mathrm{Ar}$ and $\mathrm{C}_{4} \mathrm{~F}_{8}$ gas at the input power of 0 and $500 \mathrm{~W}$, and the gas pressure of $10 \mathrm{mTorr}$. Figures 2(a) and 2(b) indicate that abnormal signals ( 0 to $10 \mathrm{amu}$ ) in the low-mass region are measured under the conditions of $500 \mathrm{~W}$, an Ar mass of $40 \mathrm{amu}$, and a carbon mass of $12 \mathrm{amu}$. The carbon mass is the smallest element that can be measured by a dissociation in $\mathrm{C}_{4} \mathrm{~F}_{8}$ plasma. Therefore, the signal measured at the low mass is not that of the elements generated by plasma. According to Medley et al., low-mass noise occurs when measuring with a quadrupole mass filter for ion beam energy over $10,000 \mathrm{eV}$ [18]. In the case of a general 


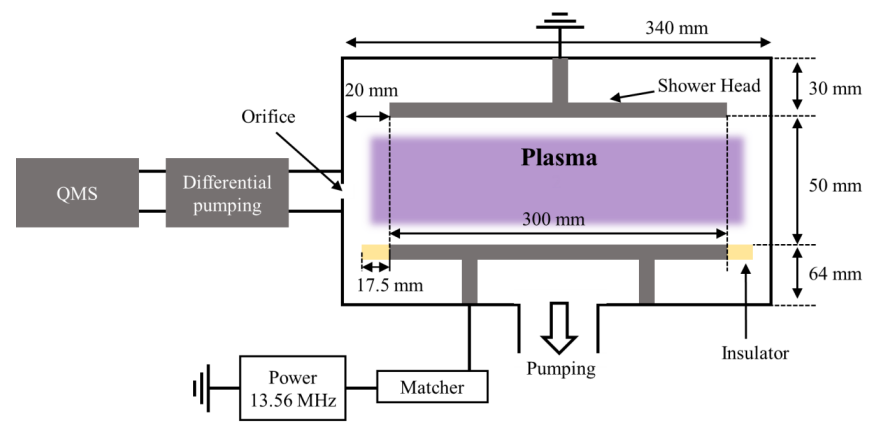

Figure 1. Schematic diagram of the experimental setup. (a)

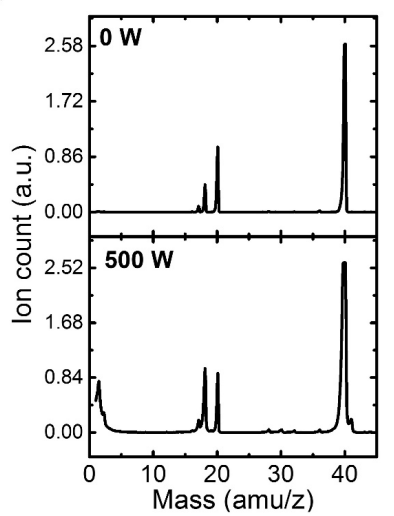

(b)

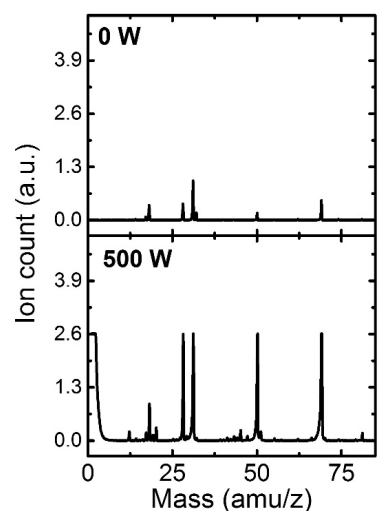

Figure 2. Comparison between mass spectra from (a) $\mathrm{Ar}$ and (b) $\mathrm{C}_{4} \mathrm{~F}_{8}$, depending on the input power conditions of 0 and $500 \mathrm{~W}$.

plasma condition, the energy of ions is in the range of tens to thousands of $\mathrm{eV}$ [19-21]. To verify whether the phenomenon is caused by ion-generated plasma, we performed a numerical analysis for QMS.

\section{Simulation details}

The QMS contained three basic elements: an ion source, a quadrupole mass filter (QMF), and an ion detector. The electrode structure of the QMF is shown in Fig. 3, where $\phi_{0}$ is the applied electrode potential, $r_{1}$ denotes the radius between the equally spaced electrodes, and $r_{2}$ is the radius of the ion beam. The voltages applied to the two electrodes located on the $x$-axis and the two electrodes located on the $y$-axis are as follows:

$$
\phi_{0}=U+V \cos (\omega t)
$$

where $U$ is the DC voltage and $V$ is the magnitude of the RF voltage. The potential in the quadrupole must satisfy Laplace's equation and is invariant in the $z$ direction in the linear quadrupole filter system. Therefore, the potential is as follows:

$$
\phi_{\mathrm{xy}}=\frac{\phi_{0}}{2 r_{1}}\left(x^{2}-\mathrm{y}^{2}\right) .
$$

The equations of motion are

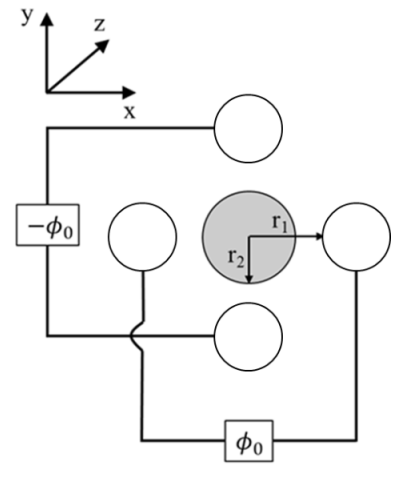

Figure 3. QMF cross-section view.

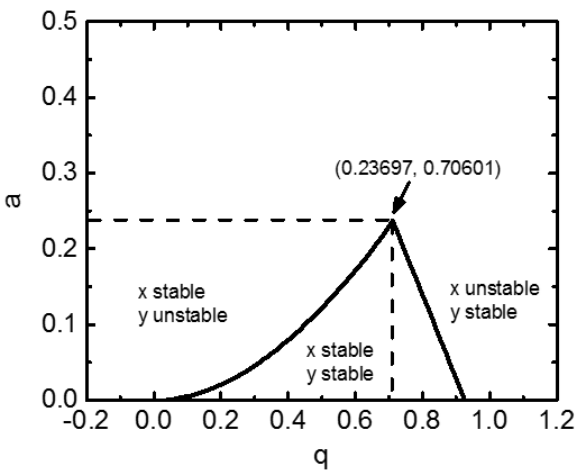

Figure 4. Schematic of the stability region in terms of dimensionless parameters a and $q$.

$$
\frac{d^{2} u}{d \xi^{2}}+\left(a_{u}-2 q_{u} \cos 2 \xi\right) u=0
$$

where $u$ is a coordinate $(x, y), \xi=\omega t, \omega=2 \pi f, \omega$ represents the angular frequency, $t$ denotes time, $f$ represents the driving frequency, and $a_{u}$ and $q_{u}$ are the stability factors. Their values are as follows:

$$
\begin{gathered}
a_{u}=a_{x}=-a_{y}=8 e U / M r_{1}^{2} \omega^{2} \\
q_{u}=q_{x}=-q_{y}=4 e V / M r_{1}^{2} \omega^{2}
\end{gathered}
$$

where $M$ is the ion mass, and $e$ is the elementary charge. The equation of motion in Eq. (4) is called Mathieu's equation.

Figure 4 shows Mathieu's first stability region for the $(a, q)$ plane, which illustrates the manner in which the filter operates. The solution of Mathieu's equation is divided into stable or unstable ion trajectories. If the conditions of $a$ and $q$, or $U$ and $V$, inside the stable region are properly selected, the ions of corresponding $M$ will have a stable trajectory. For $a$ and $q$ values outside this triangle - the condition of the ions with lower or higher masses - the ion motion is unstable either in the $x$ direction, in the $y$ direction, or in both. Therefore, the ions strike the electrodes $[8,14,22]$ simultaneously scanning the amplitudes of $U$ and $V$ along the "mass scan line," as given by [23]:

$$
U=0.167 * V
$$


(a)

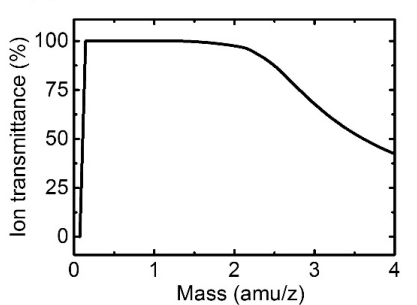

(c)

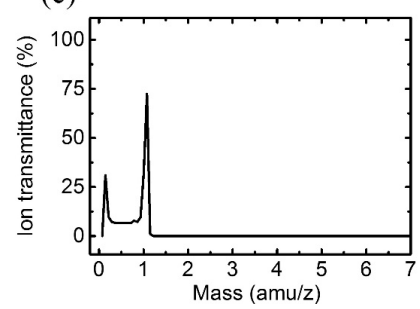

(b)

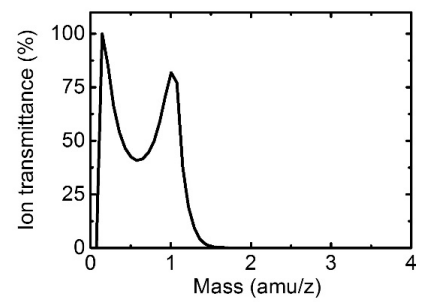

(d)

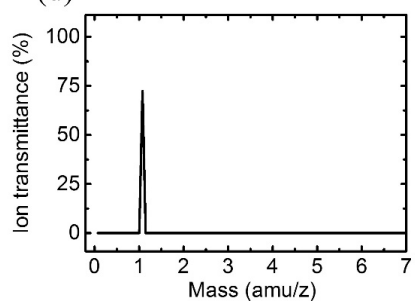

Figure 5. Mass spectra depending on the QMF residence time of the hydrogen atom: (a) $\tau_{\text {ion }}=1 \mathrm{~T}_{\mathrm{RF}}$, (b) $\tau_{\text {ion }}=5 \mathrm{~T}_{\mathrm{RF}}$, (c) $\tau_{\text {ion }}=11 \mathrm{~T}_{\mathrm{RF}}$, and (d) $\tau_{\text {ion }}=47 \mathrm{~T}_{\mathrm{RF}}$.

In this simulation, we assume that the ions are monovalent, the initial ion condition has no $x$ and $y$ velocities, and the $z$ velocity is incident at a velocity accelerated by the sheath potential. The initial ion velocity is calculated as $v_{\text {z,ion }}=\sqrt{\frac{2 q V_{a}}{M}}$, where $V_{a}$ is the acceleration voltage. The structure of the QMF calculated in the simulation is $0.1143 \mathrm{~m}$ in length, the applied frequency is $1 \mathrm{MHz}, r_{1}=0.01 \mathrm{~m}$, and $r_{2}$ $=0.005 \mathrm{~m}$. For the structure information, the QMS structure was used in the experiment. In the case of the voltage-to-mass conversion, the $q$ value at the peak of the stability region was converted using the following:

$$
M=\left(\frac{2 e}{0.70601 \omega^{2} r_{0}^{2}}\right) V
$$

In this simulation, Eq. (3) is calculated in two steps using the Euler method [24]. First, the velocity term is given by

$$
\frac{d u}{d t}\left(t_{i+1}\right)=\frac{d u}{d t}\left(t_{i}\right)+\Delta t \frac{d}{d t}\left(\frac{d u}{d t}\right)\left(t_{i}\right)
$$

Then, the position term is given by

$$
u\left(t_{i+1}\right)=u\left(t_{i}\right)+\Delta t \frac{d u}{d t}\left(t_{i}\right)
$$

where $u\left(t_{i}\right)$ is the displacement of the ion at time $t_{i}$, and $\Delta t$ is the time step, $t_{i+1}-t_{i}$. In the above calculation, $\Delta t$ is set to $1 / 100$ of the RF period, and the initial position is distributed with the same distance of 100,000 ions in the ion beam of $r_{2}$. Moreover, the collision between the incoming particles is neglected in this simulation because the pressure inside the QMS is below the $10^{-5}$ Torr condition; consequently, the mean free path of the particles is long [20].

Figure 5 depicts the ion transmittance of the hydrogen ions (1 $\mathrm{amu}$ ) in accordance with the ion velocity. If the ion passing time of the quadrupole mass filter $\left(\tau_{\text {ion }}\right)$ is one cycle of the RF period $\left(1 \mathrm{~T}_{\mathrm{RF}}\right)$, all

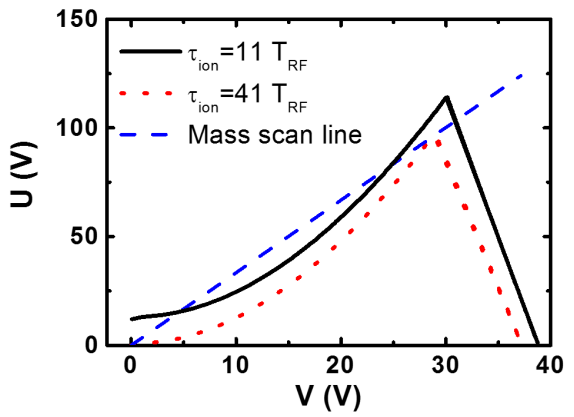

Figure 6. Stability region depending on the QMF residence time of the hydrogen atom.

(a)

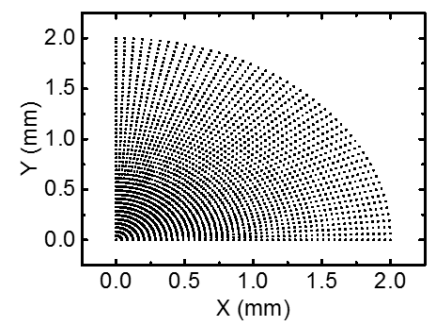

(b)

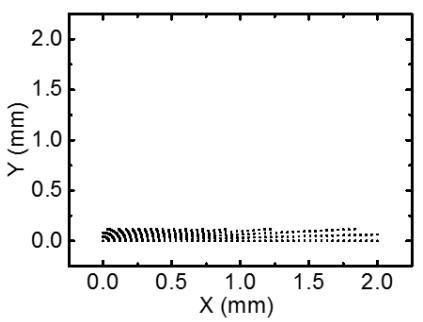

(c)

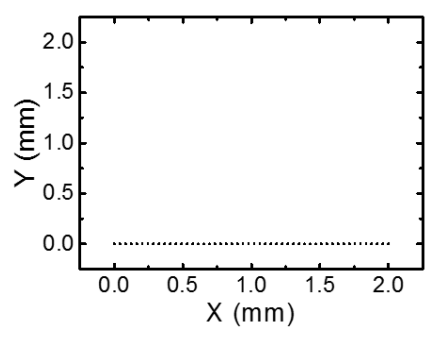

Figure 7. QMF transmission characteristics of the ion beam depending on the QMF residence time of the hydrogen atom: (a) $\tau_{\text {ion }}=1 \mathrm{~T}_{\mathrm{RF}}$, (b) $\tau_{\text {ion }}=7 \mathrm{~T}_{\mathrm{RF}}$, and (c) $\tau_{\text {ion }}=$ $27 \mathrm{~T}_{\mathrm{RF}}$ at the filter voltage of $U=15 \mathrm{~V}, V=5 \mathrm{~V}$ condition.

hydrogen ions pass through in 0 to $2 \mathrm{amu}$, and an average of $72 \%$ of the ions passes through in 2 to $4 \mathrm{amu}$. This phenomenon decreases as the speed of the ions decreases, as shown in Figs. 5(b) and 5(c). As the $\tau_{\mathrm{ion}}=47 \mathrm{~T}_{\mathrm{RF}}$ condition, the hydrogen atoms are filtered in the mass area except for $1 \mathrm{amu}$. In the case of plasma, it is thought that this phenomenon may occur because high-energy ions enter QMS on account of acceleration in the sheath potential [20]. This can be also confirmed in the stability region, as shown in Fig. 6. If the filter pass time of the ions is short, it can be observed that there is a point overlapping the scan line in the small RF and DC voltage regions. This means that ions reach the detector before being filtered in response to the quadrupole electric field.

Figure 7 shows the ions passing through each initial ion position according to the filter pass time at the filter voltage of $U=15 \mathrm{~V}$ and the $V=5 \mathrm{~V}$ condition. The corresponding filter voltage condition is that in which $1 \mathrm{amu}$ of ions is filtered. When the $\tau_{\text {ion }}=1 \mathrm{~T}_{\mathrm{RF}}$ condition exists [Fig. 7(a)], no ions passing through the quadrupole filter are filtered. When $\tau_{\text {ion }}$ increases, as shown Fig. $7(\mathrm{~b})$, the ions located far from the $x$-axis are filtered. Moreover, under the $\tau_{\text {ion }}=27 \mathrm{~T}_{\mathrm{RF}}$ condition, 
(a)

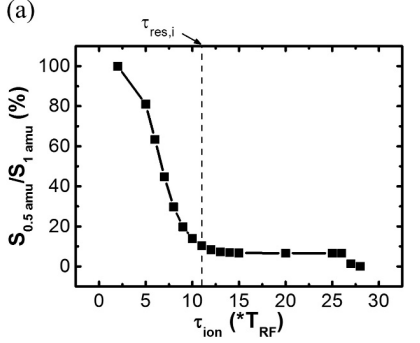

(b)

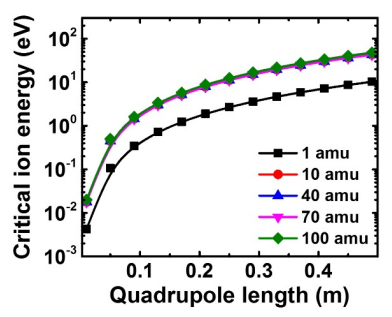

Figure 8. (a) Signal ratio of 0.5 and 1 amu for $\tau_{\text {ion }}$ of the hydrogen atom. (b) Required QMF length for ion energy of various masses.

the ions are filtered in the region except for those located in the $x$-axis, as shown in Fig. 7(c). The reason that the particles located on the $x$-axis are not well filtered is because the DC voltage applied to the $x$-axis rod is positive, and the movement of the cation in the $x$-axis rod direction is considered limited.

To effectively remove this low mass noise, it is necessary to adjust the QMF length with consideration of the $\tau_{\text {ion }}$ and the radius of the incident ion beam. Figure 8 shows the signal ratio of the hydrogen atom to the low-mass noise according to $\tau_{\text {ion }}$ for the hydrogen atom. It additionally depicts the required QMF length for the ion energy of various masses for 1 to $100 \mathrm{amu}$ atoms. For quadrupole mass spectrometers, the low-mass noise and mass signal can be distinguished if the signal at $0.5 \mathrm{amu}$ is reduced to less than $10 \%$. This is because the mass resolution is usually less than one $[4,9]$. Therefore, we assume that the point at which the transmittance at $0.5 \mathrm{amu}$ becomes $10 \%$ or less of the transmittance at the hydrogen atom mass is the point at which low-mass noise disappears. In Fig. 8(a), it is observed that the ratio of $1 \mathrm{amu}$ signal $\left(S_{\text {lamu }}\right)$ to the low-mass noise signal $\left(S_{0.5 \mathrm{amu}}\right)$ is reduced to less than $10 \%$ when $\tau_{\text {ion }}>11 \mathrm{~T}_{\mathrm{RF}}$.

Therefore, to measure the hydrogen ions in the plasma, the length of the quadrupole filter must satisfy the $\tau_{\text {ion }}>11 \mathrm{~T}_{\mathrm{RF}}$ condition to exclude the low-mass noise. In addition, $\tau_{\mathrm{ion}}=11 \mathrm{~T}_{\mathrm{RF}}$ is defined as $\tau_{\text {res, } i,}$ the allowable energy of hydrogen ions that can be obtained at a given QMF length. This is given by $\frac{1}{2} M\left(l / \tau_{\text {res, } i}\right)^{2}=q E_{c i}$, where $l$ is the QMF length, $q$ denotes the electric charge quantity, and $E_{c i}$ is the critical ion energy, that is, the energy of the ion that satisfies $S_{0.5 \mathrm{amu}} /$ $S_{\text {mass }}<10 \%$, where $S_{\text {mass }}$ is the signal of the incident ion.

Figure 8(b) shows $E_{c i}$ depending on the QMF length for the various masses. If the $E_{i}>E_{c i}$ condition exists, low-mass noise can affect the radical measurement in low-pressure plasmas. If the $E_{i}>E_{c i}$ condition exists, it can be expected that a measurement without noise is possible. For example, if the QMF length is small $(<0.2 \mathrm{~m})$, a lowmass signal may be generated, even by ions of $10 \mathrm{eV}$ or less, and the influence of ions of $10 \mathrm{amu}$ or less is more prominent. Therefore, in plasma measurement under a high ion energy and high voltage sheath, the QMF length must be set as long, or a retarding field must be used to control the ion energy [25].

Figure 9 shows the normalized signal ratio of the original mass to noise for particles with 1,40 , and $70 \mathrm{amu}$, as well as the radius of the ion beam. In the mass region of 1 to $70 \mathrm{amu}$, low mass noise is effectively removed when the beam radius is 0.2 times the filter system radius $\left(r_{1}\right)$. If the beam diameter is less than 0.2 , the noise signal is relatively large because there are relatively many particles near the $x$-axis. Moreover, if the radius of the beam is larger than 0.2 times $r_{1}$,

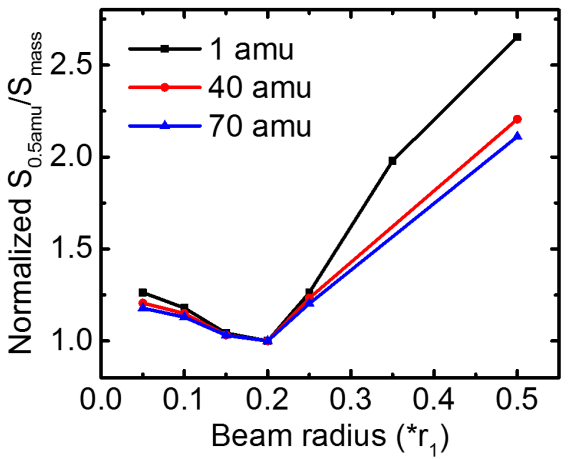

Figure 9. Normalized noise signal ratio of 1,40 , and 70 amu compared to $0.5 \mathrm{amu}$, depending on the beam radius.

the noise signal is small; however, the signal at the original mass is also small. Thus, the efficiency of the mass filtering is poor.

\section{Conclusions}

In this study, we analyzed the low-mass noise that can occur when measuring plasma using a quadrupole mass spectrometer. We confirmed that the abnormal signal can be caused by ions accelerated by the sheath potential. In addition, we confirmed that if the filter length is small, it can be caused by a small potential. To solve this issue, we verified that the intensity of the abnormal signal can be reduced by considering the filter length for ion energy and the diameter of the incident ion beam.

\section{Acknowledgements}

This research was supported by a National Research Council of Science \& Technology (NST) grant by the Korea government (MSIP) (No. CAP-17-02-NFRI), by the Korea Institute of Energy Technology Evaluation and Planning (KETEP), and the MOTIE of the Republic of Korea (No. 20172010105910). In addition, it was supported by a Korea Institute for Advancement of Technology (KIAT) grant funded by the Korea Government (MOTIE) (P0008458, The Competency Development Program for Industry Specialist), by the Aerospace Low Observable Technology Laboratory Program of the Defense Acquisition Program Administration and the Agency for Defense Development of the Republic of Korea, and by the Basic Science Research Program through the National Research Foundation of Korea (NRF) funded by the Ministry of Education (NRF-2020R1A6A1A03047771).

\section{References}

[1] H. C. Lee, Appl. Phys. Rev. 5, 011108 (2018).

[2] F. Hernández, J. V. Sancho, M. Ibáñez, E. Abad, T. Portolés, and L. Mattioli, Anal. Bioanal. Chem. 403, 1251 (2012).

[3] H. Kojima, H. Sugai, T. Mori, H. Toyoda, and T. Okuda, J. Nucl. Mater. 128-129, 965 (1984).

[4] R. E. March, R. J. Hughes, and A. D. Roberts, Anal. Chim. Acta 243, 323 (1991).

[5] H. D. Kang and V. Dose, J. Vac. Sci. Technol. A Vacuum, Surfaces, Film. 21, 1978 (2003).

[6] C. Ding, N. V. Konenkov, and D. J. Douglas, Rapid Commun. 
Mass Spectrom. 17, 2495 (2003).

[7] R. K. Julian, H. Reiser, and R. Graham Cooks, Int. J. Mass Spectrom. Ion Process. 123, 85 (1993).

[8] M. Henchman and C. Steel, J. Chem. Educ. 75, 1049 (1998).

[9] G. L. Glish and R. W. Vachet, Nat. Rev. Drug Discov. 2, 140 (2003).

[10] R. S. Gohlke, Anal. Chem. 31, 535 (1959).

[11] M. Guo, D. Li, Y. Cheng, Y. Wang, W. Sun, X. Pei, M. Dong, X. Sheng, L. Zhao, and Y. Li, Eur. J. Mass Spectrom. 24, 206 (2018).

[12] M. Schuhmacher, B. Rasser, E. De Chambost, F. Hillion, T. Mootz, and H. N. Migeon, J. Anal. Chem. 365, 12 (1999).

[13] P. H. Dawson, Quadrupole Mass Spectrometry and its Applications (Elsevier, 1976).

[14] K. Blaum, C. Geppert, and P. Mu, Int. J. Mass Spectrom. 181, 67 (1998).

[15] F. Zhuge, W. Dai, C. L. He, A. Y. Wang, Y. W. Liu, M. Li, Y. H. Wu, P. Cui, and R. W. Li, Appl. Phys. Lett. 96, 163505 (2010).
[16] P. H. Dawson, Int. J. Mass Spectrom. Ion Phys. 17, 423 (1975).

[17] P. H. Dawson, Int. J. Mass Spectrom. Ion Phys. 20, 237 (1976).

[18] S. S. Medley, Rev. Sci. Instrum. 49, 698 (1978).

[19] D. Choi, H. J. Yeom, K. H. You, J. H. Kim, D. J. Seong, E. Yoon, and H. C. Lee, Carbon 162, 423 (2020).

[20] M. A. Lieberman and A. J. Lichtenberg, Principles of Plasma Discharges and Materials Processing, 2nd Ed. (John Wiley \& Sons, Inc., 2005).

[21] E. A. Edelberg and E. S. Aydil, J. Appl. Phys. 86, 4799 (1999).

[22] J. E. Campana, Int. J. Mass Spectrom. Ion Phys. 33, 101 (1980).

[23] J. R. Gibson, S. Taylor, and J. H. Leck, J. Vac. Sci. Technol. A Vacuum, Surfaces, Film 18, 237 (2000).

[24] C. W. Gear and L. R. Petzold, SIAM J. Numer. Anal. 21, 716 (1984).

[25] D. Gahan, B. Dolinaj, and M. B. Hopkins, Rev. Sci. Instrum. 79, 033502 (2008). 